\title{
DETERMINATION OF MOISTURE CONTENT IN SYNTHETIC MOULDING SAND ON THE GROUNDS OF RELATIVE PERMITTIVITY MEASUREMENT
}

\begin{abstract}
The presented research was aimed at searching for an exact and effective method of determining moisture content in traditional moulding sands. By measuring resonance frequency and quality factor of a waveguide resonance cavity, relative permittivity was determined for different synthetic moulding sands. Analysis of the presented results confirms a linear relation between relative permittivity values and moisture content values in the selected traditional moulding sands. The obtained linear relationship can be used as a reference characteristic for evaluation of humidity of moulding sand.
\end{abstract}

Keywords: moisture content, moulding sands, relative permittivity, microwaves

\section{Introduction}

In synthetic moulding sands classified as $1^{\text {st }}$ generation sandmixes, water is a component that ensures suitable technological properties, in particular strength, permeability and friability. In foundry processes, exact knowledge of moisture content in moulding sand is important, since its change by $0.5 \%$ only results in significantly worsened quality of moulding sand and thus of the obtained castings. For this reason, moisture content - as one of the factors deciding on the quality of moulding sands - should be continuously controlled [1].

Most of the commonly known and applied methods of measuring moisture content in traditional moulding sands have some disadvantages and restrictions, which include long measuring time and low accuracy [2]. So, it is well-grounded to search for such a method of measuring moisture content that would be characterised by short measuring time and high accuracy. A method that meets these requirements was developed in the Department of Foundry Engineering, Plastics and Automation of Wroclaw University of Science and Technology. In this new method, moisture content in a moulding sand is determined on the grounds of known permittivity $\varepsilon_{r}$ determined by the perturbation method on a stand of waveguide resonance cavity shown in Fig. 1.

Relative permittivity of a dielectric material characterises its ability to be polarized in microwave field with a given frequency. In many scientific works it has been shown that a value of permittivity depends, among the others, on microwave field frequency, qualitative and quantitative composition of the material, and especially on the content of water that is the main absorber of

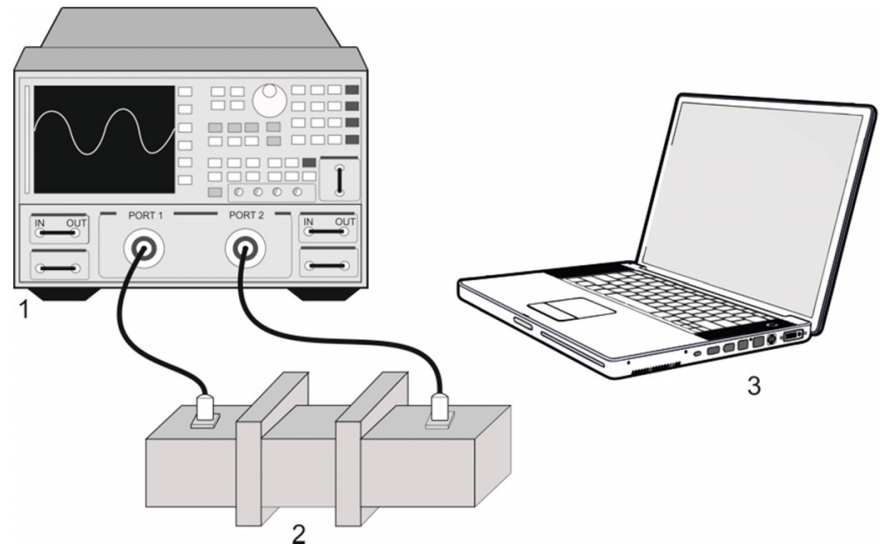

Fig. 1. Stand of waveguide resonance cavity: 1 - vector network analyzer, 2 - waveguide resonance cavity, 3 - computer

electromagnetic radiation [3-11]. Till now, the results presented in the matter literature have been related to quartz sand or soil that, like moulding sand, is a multiphase mixture. In the foundry theory or practice, there is no study that would consider humidity measurement of traditional moulding sands on the grounds of their known relative permittivity. The presented work is aimed at searching for an exact and effective method of determining moisture content in traditional moulding sands.

Relative permittivity of the material constitutes a dimensionless quantity that determines how many times permittivity of the given medium $\varepsilon\left(\mathrm{F} \cdot \mathrm{m}^{-1}\right)$ is lower than the permittivity of vacuum $\varepsilon_{0}\left(\mathrm{~F} \cdot \mathrm{m}^{-1}\right)$, as shown by Eq. (1).

$$
\varepsilon_{r}=\frac{\varepsilon}{\varepsilon_{0}}
$$

* WROCŁAW UNIVERSITY OF SCIENCE AND TECHNOLOGY, WYBRZEŻE STANISŁAWA WYSPIAŃSKIEGO 27, 50-370 WROCŁAW, POLAND

\footnotetext{
Corresponding author: daniel.nowak@pwr.edu.pl
} 
Relative permittivity $\varepsilon_{r}$ is determined for dielectric materials only, which become polarized under the action of external electric field. Then, electric charges appear on the surface of a real dielectric, creating electric field with a phase shift $\delta$ and opposite direction in relation to the field that causes polarization.

Depending on the mechanism of polarization under the electric field and type of the polarized molecules, the following basic kinds of polarization are distinguished [12]:

- Electronic polarization occurs when negatively charged clouds of electrons are displaced with respect to positively charged nuclei. It occurs in all materials. This is a rapid process, observed even at variable currents corresponding to ultraviolet radiation.

- Atomic polarization, called also molecular polarization, occurs when differently charged atoms are displaced with respect to each other in a molecule. The mass of atoms is much larger that that of electrons, so the reaction to a change of electric field is slower. The phenomena resulting from the atomic polarization are observed till the frequency corresponding to infrared radiation. Its part in total polarization is much smaller than that of electronic polarization, so it is sometimes disregarded.

- Ionic polarization occurs in the materials with ionic bonds. It is caused by displacement of differently charged ions in crystal lattice of the material. It is the slowest of all the polarization processes, since it requires movements of numerous ions bounded in the lattice.

- Orientation or dipolar polarization occurs in polar dielectrics i.e. those where molecules create durable dipoles (have their own dipole moment). In the electric field, they are subjected to the action of an ordering moment of force. At the same time, the order is destroyed by thermal vibrations which causes that this kind of polarization is temperaturedependent.

- Polarization by spatial charges occurs in the materials with macroscopic areas in which the charge carriers can move but their freedom is for some reasons limited to the areas smaller than size of the body. They can be i.e. conducing granules arranged in insulating matrix. The charges can move within conducing phase grains that, in that way, become dipoles.

Macroscopic properties of dielectrics in variable electric field are described by relative complex permittivity $\varepsilon_{r}$ determined by the Eq. (2). It should be noted that this property is not a material constant since it changes in relation to both electrical parameters of the materials and the frequency of electromagnetic wave.

$$
\varepsilon_{r}=\varepsilon^{\prime}-j \varepsilon^{\prime \prime}
$$

where:

$\varepsilon^{\prime}$ - real component of relative complex permittivity,

$\varepsilon^{\prime \prime}$ - imaginary component of relative complex permittivity.

The real component of relative complex permittivity expresses the ability of the material to accumulate energy, and the imaginary component is responsible for its ability to disperse the energy accumulated in the electric field, i.e. to generate losses [13].

Permittivity of dielectric materials depends on temperature, the intensity of electric field and humidity. The relationship between permittivity and humidity was used for developing a new method of measuring moisture content in traditional moulding sands.

\section{Methodology and test stand}

As the base of the examined moulding sands, the most commonly used and commercially available high-silica sand was applied, coming from two different deposits in Poland, i.e. from the mines "Szczakowa” S.A. and „Grudzeń Las” Ltd. In traditional moulding sands, refractory clay is most often used as the binder. In the presented research, three kinds of foundry bentonites with different technological properties were applied, with the trade names Monobent Extra, Geko and Specjal [14]. Quantitative and qualitative compositions of the examined traditional moulding sands are given in Table 1 . To optimise humidity of moulding sands, concentration of water was selected in the range between $1 \%$ and $6 \%$.

TABLE 1

Quantitative and qualitative compositions of moulding sands

\begin{tabular}{|c|c|c|}
\hline $\begin{array}{c}\text { Sandmix } \\
\text { No. }\end{array}$ & Quartz sand base & Binding material \\
\hline 1 & Grudzeń Las medium $-95 \%$ & Monobent Extra $-5 \%$ \\
\hline 2 & Grudzeń Las medium $-89 \%$ & Monobent Extra $-11 \%$ \\
\hline 3 & Szczakowa medium $-95 \%$ & Monobent Extra $-5 \%$ \\
\hline 4 & Szczakowa medium $-89 \%$ & Monobent Extra $-11 \%$ \\
\hline 5 & Grudzeń Las medium $-95 \%$ & Geko $-5 \%$ \\
\hline 6 & Grudzeń Las medium $-95 \%$ & Specjal $-5 \%$ \\
\hline
\end{tabular}

The moulding sands were prepared in accordance to literature recommendations [2]. For the examinations, cylindrical specimens dia. $16 \mathrm{~mm}$ and $43 \mathrm{~mm}$ high were prepared. It was shown in [15] that relative permittivity of traditional moulding sands depends on their apparent density. For this reason, on the grounds of mass and volume measurements of each of the compacted specimens, apparent density that ranged between 1420 and $1580 \mathrm{~kg} / \mathrm{m}^{3}$ was determined. Moisture content in individual moulding sands was determined by the gravimetric method on 50 -g specimens which was being dried for 15 minutes.

Measurements of relative permittivity $\varepsilon_{r}$ are carried-out by various selected methods depending on the kind of a material, size and shape of the specimens, electric field frequency and anticipated ranges of the measured values. In this research, the perturbation method was chosen from among many methods of measuring relative permittivity of traditional moulding sands. It permits measurements in wide ranges of temperature and humidity, as well as precise determination of complex value $\varepsilon_{r}$ at the industrially used frequency $2.45 \mathrm{GHz}$. Measurement time for one specimen was ca. $45 \mathrm{~s}$. 
Detailed description of the measurement methodology and the relationships making grounds for permittivity calculations are given in [16]. Measurements were taken at $20^{\circ} \mathrm{C}$ and relative air humidity of $40 \%$. The results were analysed in an integrated system for statistical data analysis.

\section{Results and discussion}

Measured values of relative permittivity $\varepsilon_{r}$ for selected moulding sands in relation to their water (moisture) content are shown in Figs. 2-7. The determined relationships $\varepsilon_{r}=f(W)$ are drawn in the figures in a form of regression lines. For all the examined moulding sands, Pearson's linear correlation coefficients $r$ were determined, as well as confidence intervals for the confidence level $1-\alpha=0.95$, which limits are marked in the figures by broken lines.

The analysis of the presented results indicates that, in each of the examined moulding sands, increase of moisture content results in increased value of relative permittivity. For example, increase of water content in the sandmix No. 5 from $2.3 \%$ to $5.63 \%$ results in 1.5 -fold increase of the $\varepsilon_{r}$ value. A similar in-

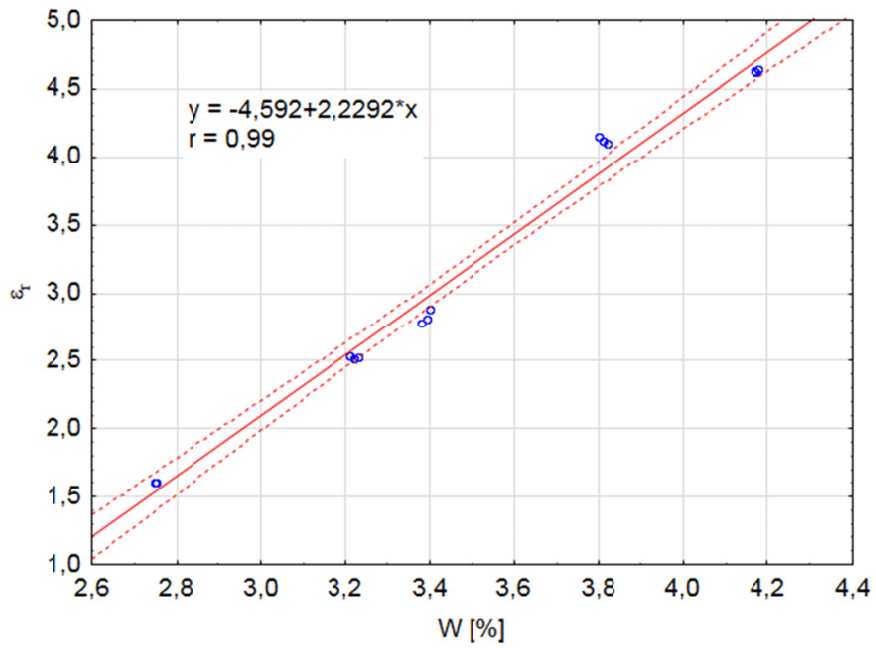

Fig. 2. Relationship $\varepsilon_{r}=f(W)$ for the sandmix No. 1

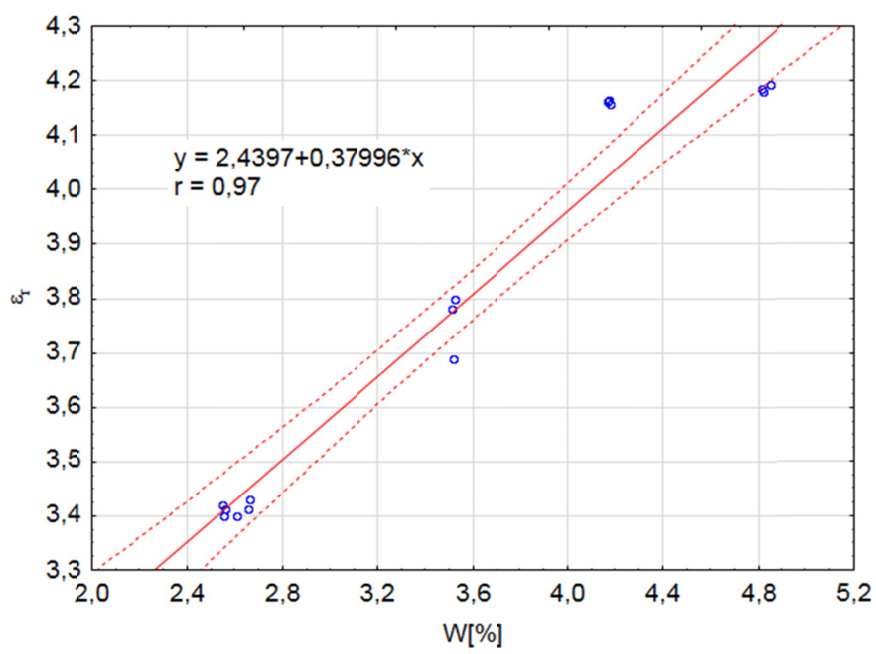

Fig. 4. Relationship $\varepsilon_{r}=f(W)$ for the sandmix No. 3 crease of relative permittivity value is observed for the sandmix No. 4 as a result of 4 -fold increase of water content.

This is due to the fact that by examining the dielectric constant multiphase mixtures in which one of the ingredients is necessary to take its high value $\varepsilon_{r}$ into consideration which at room temperature is $\varepsilon_{r}=81$. A water molecule as a result of asymmetric spatial distribution of charges has a clear polarity which results in extremely high dipole moment. This feature makes the water molecules interact electrically both with each other and with the molecules of other substances which are in direct contact with water [8]. The conducted study reveals that an increase in the water content in the tested molding sand at the same time causes a linear increase of dielectric permittivity of the studied molding sand as it is seen on (Figs. 2-7) in the moulding sands humidity ranging from 1.3 to $5.6 \%$.

The performed statistical analysis that gives positive values of linear correlation coefficients within 0.97 to 0.99 confirms a very strong linear relation between $\varepsilon_{r}$ values and moisture content values $\mathrm{W}$ in the selected traditional moulding sands.

The $95 \%$ confidence intervals are quite narrow, which shows that the $\varepsilon_{r}$ values in relation to moisture content W were obtained with correct and proper accuracy of measurements.

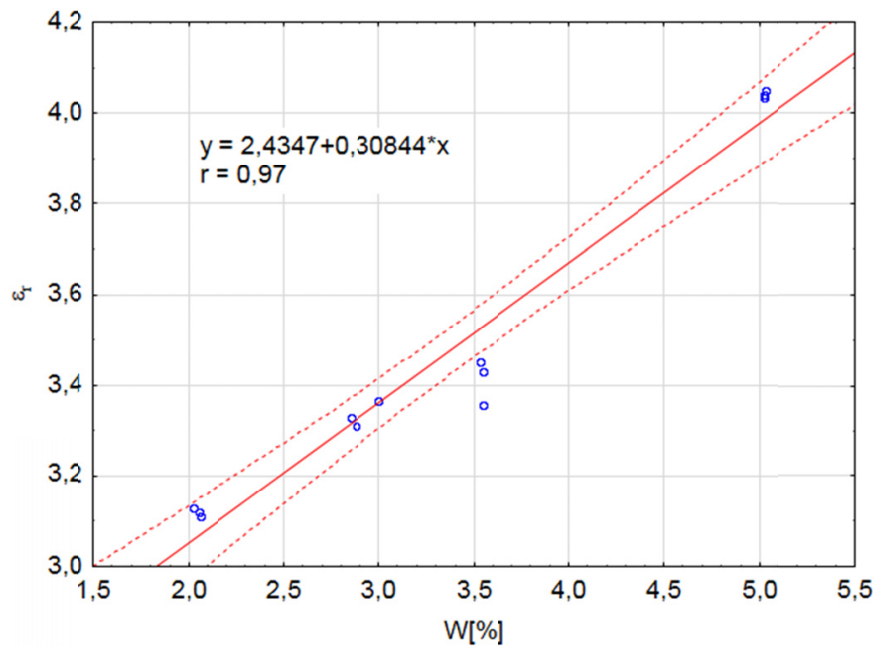

Fig. 3. Relationship $\varepsilon_{r}=f(W)$ for the sandmix No. 2

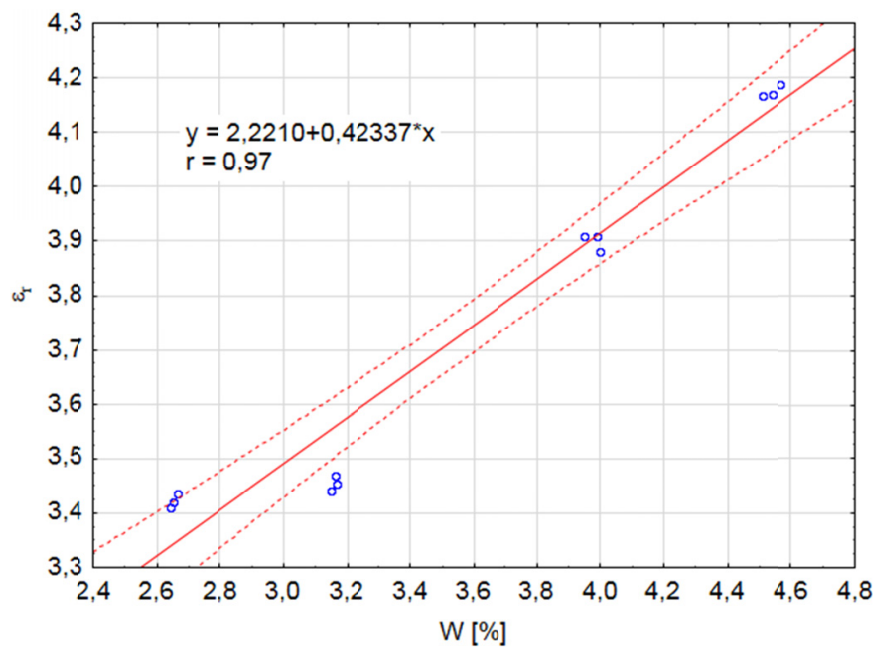

Fig. 5. Relationship $\varepsilon_{r}=f(W)$ for the sandmix No. 4 


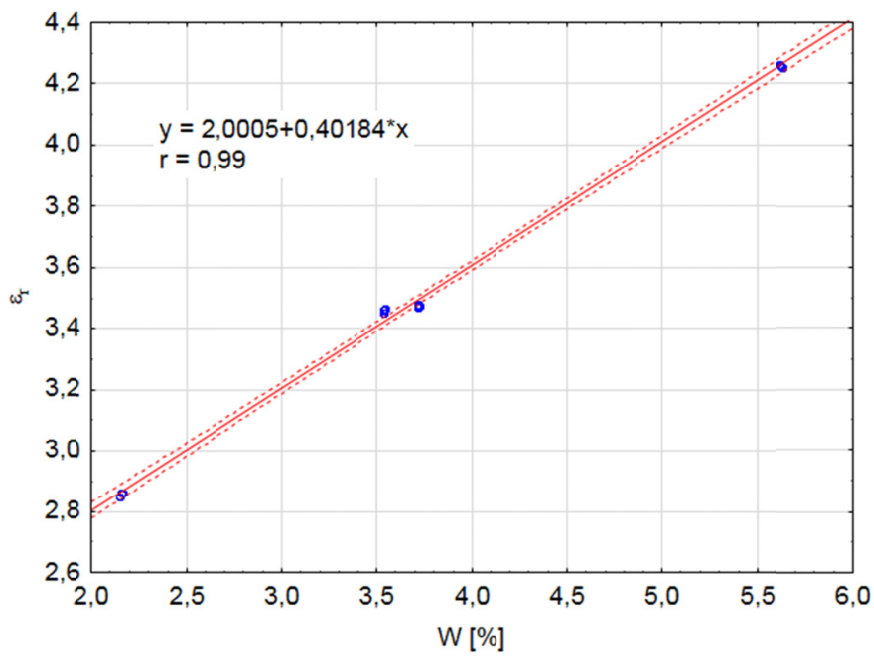

Fig. 6. Relationship $\varepsilon_{r}=f(W)$ for the sandmix No. 5

For each of the examined sandmixes, a reference characteristic $\varepsilon_{r}=f(W)$ can be determined from the function of linear regression that describes the relation between permittivity and water content, on the grounds of a few measuring points only.

\section{Conclusions}

One of the crucial parameters describing the electrical properties of the material centers is the dielectric permittivity. This property has also been used to describe multiphase materials and a conventional moulding sand can be treated as such. The influence of moulding sand humidity on the dielectric permittivity was particularly studied. It is important to know the parameter measurement result, which is the mass moisture, while the production of high quality castings. Therefore, researches on new methods of testing for moisture content in moulding sands become important. At work a new method of determining moisture content in traditional moulding sands is presented. The analysis of measurement results shows that the obtained linear relationship between permittivity and water content in a moulding sand can be used as a reference characteristic for evaluation of its humidity. The developed method is characterised by short measuring time of ca. $45 \mathrm{~s}$ and high accuracy. There is also the possibility to automate the measuring process which would result in further reduction of measuring time.

\section{REFERENCES}

[1] S.M. Dobosz, Water in moulding and core sands, 2006 Publishing House Akapit, Kraków.

[2] L. Lewandowski, Materials for casting moulds, 1997 Publishing House Akapit, Kraków.

[3] J. Wang, T. Schmugge, D. Williams, Dielectric constants of soils at microwave frequencies-II, National Aeronautics and Space Administration, NASA Tech. Pap. 1238, May 1978.

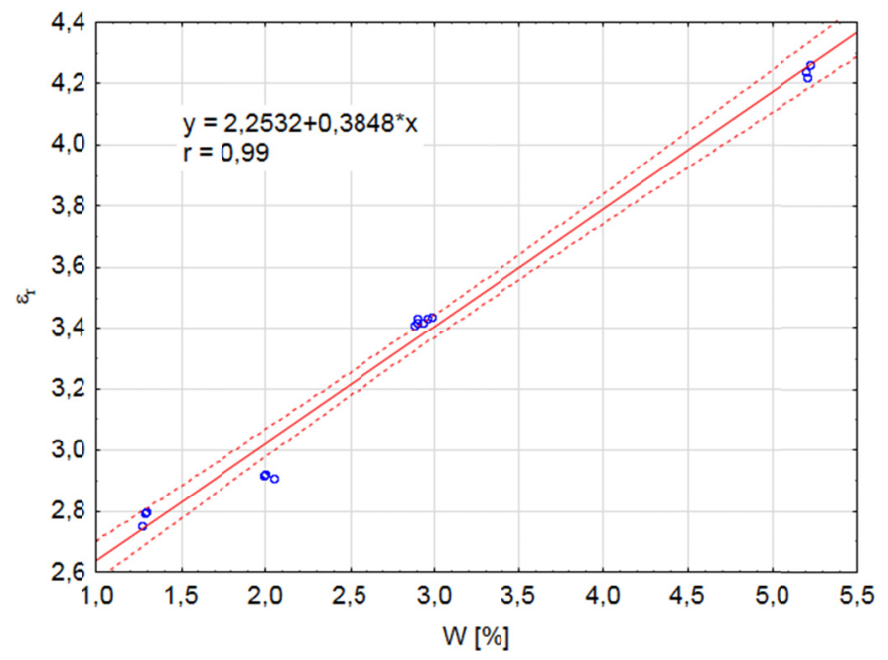

Fig. 7. Relationship $\varepsilon_{r}=f(W)$ for the sandmix No. 6

[4] J.E. Hipp, Soil electromagnetic parameters as a function of frequency, soil density and soil moisture, Proc. IEEE, 62, 98-103 (1974).

[5] G.P. de Loor, Dielectric properties of heterogeneous mixtures containing water, J. Microwave Power 3, 67-73 (1968).

[6] C. Liu, L. Zhang, J. Peng, C. Srinivasakannan, B. Liu, H. Xia, J. Zhou, L. Xu, Temperature and moisture dependence of the dielectric properties of silica sand, J. Microwave Power Ee. 47 (3), 199-209 (2013).

[7] X. Shang, J. Chen, W. Zhang, J. Peng, H. Chen, S. Guo, G. Chen, Dimension Optimization for Silica Sand Based on the Analysis of Dynamic Absorption Efficiency in Microwave, Dry. Technol. 32, 1608-1613 (2014).

[8] L.M. Dudley, S. Bialkowski, D. Or, C. Junkermeier, Low frequency impedance behavior of montmorillonite suspensions, Soil Sci. Soc. Amer. J. 67 (2), 518-526 (2003).

[9] A. Revil, Effective conductivity and permittivity of unsaturated porous materials in the frequency range $1 \mathrm{mHz}-1 \mathrm{GHz}$, Water Resources Res. 49 (1), 306-327 (2013).

[10] T.C. Baum, K. Ghorbani, Measurements on the Effects of Moisture on the Complex Permittivity of High Temperature Ash, Microwave Theory and Techniques IEEE Transactionson 64, 607-615 (2016).

[11] P.W. Rosenkranz, A model for the complex dielectric constant of supercooled liquid water at microwave frequencies, IEEE Trans. Geosci. Remote Sens. 53 (3), 1387-1393 (2015).

[12] R. Drożdzak, K. Twardowski, Permittivity of porous media factors affecting its variability, Drilling Oil Gas 27, 1-2 (2010).

[13] M. Lisowski, Measurements of resistivity and permittivity of solid dielectrics, 2004 Publishing House of Wrocław University of Technology, Wrocław.

[14] M. Holtzer, A. Bobrowski, B. Grabowska, Montmorillonite: a comparison of methods for its determination in foundry bentonites, Metalurgija 50 (2), 119-122 (2011).

[15] D. Nowak, K. Granat, B. Opyd, Examination and analysis of influence of compaction degree on dielectric properties of moulding sand components, Metalurgija 54 (2), 353-356 (2015).

[16] K. Granat, B. Opyd, D. Nowak, M. Stachowicz, G. Jaworski, Usefulness of foundry tooling materials in microwave heating process, Arch. Metall. Mater. 58 (3), 919-922 (2013). 